\title{
Identifying novel targets of resveratrol
}

Resveratrol is thought to mimic the effects of caloric restriction to confer anti-ageing and antidiabetogenic properties, but its mechanism of action has remained controversial. Chung and colleagues now report that resveratrol increases cyclic AMP levels by competitively inhibiting phosphodiesterases (PDEs), ultimately leading to the activation of AMP-activated protein kinase (AMPK) and positive metabolic effects.

Chung and his team had previously shown that AMPK was a key mediator of the metabolic effects of resveratrol but not a direct target of this polyphenol. In the present study, published in Cell, they confirmed a previously reported increase in cAMP levels in response to resveratrol, before showing that inhibition of adenylyl cyclase prevented AMPK phosphorylation. They then demonstrated that the CAMP-regulated guanine nucleotide exchange factor EPAC1 was required for AMPK activation in HeLa cells, and that resveratrol indirectly increased EPAC1 activity. EPAC1 was also required for the resveratrol-mediated increase in the levels of $\mathrm{NAD}^{+}$and the activity of the sirtuin 1 deacetylase.

Calcium/calmodulin-dependent kinase kinase- $\beta$ (CaMKK $\beta$ ) and liver kinase B1 (LKB1) are two upstream kinases that phosphorylate and thereby activate AMPK. EPAC1 can increase cytosolic $\mathrm{Ca}^{2+}$ levels via CaMKK $\beta$ in a phospholipase $\mathrm{C}$ (PLC)-dependent manner, and treatment with a calcium chelator, a CaMKK $\beta$ inhibitor or a PLC inhibitor decreased AMPK phosphorylation in response to resveratrol. EPAC1 also induces calcium release from internal stores via ryanodine receptor 2 (RYR2), and Chung's team showed that resveratrol required active EPAC1 to activate RYR2. So, resveratrol, via the EPAC1-PLCRYR2 pathway, promotes CaMKK $\beta$ mediated activation of AMPK. The authors point out that, depending on the condition and the cell type, the alternative cAMP effector protein kinase A might also be involved in resveratrol-mediated activation of AMPK by activating LKB1.

The key issue, however, is how resveratrol raises cAMP levels. Chung and colleagues showed that it did not stimulate the activity of adenylyl cyclases, prompting them to investigate potential inhibitory effects on the CAMP-selective PDEs. Resveratrol inhibited the activity of PDE1, PDE3 and PDE4 by competing with cAMP for PDE binding.

Given these results, PDE inhibitors would be expected to mimic the metabolic effects of resveratrol, and so - on the basis that PDE4 is responsible for most PDE activity in myotubes and skeletal muscles - Chung's team treated these cells with rolipram, a PDE4 inhibitor. Like resveratrol, rolipram increased cAMP levels and NAD ${ }^{+}$levels, stimulated AMPK phosphorylation and induced deacetylation of a sirtuin 1 substrate. Mice that were fed a high-fat diet and treated with rolipram exhibited increased mRNA levels of genes known to be induced by resveratrol, as well as enhanced mitochondrial biogenesis. The mice were also resistant to weight gain, which resulted from an increased basal metabolic rate. Furthermore, as expected from the metabolic changes induced by rolipram, treatment with this inhibitor increased glucose tolerance compared with

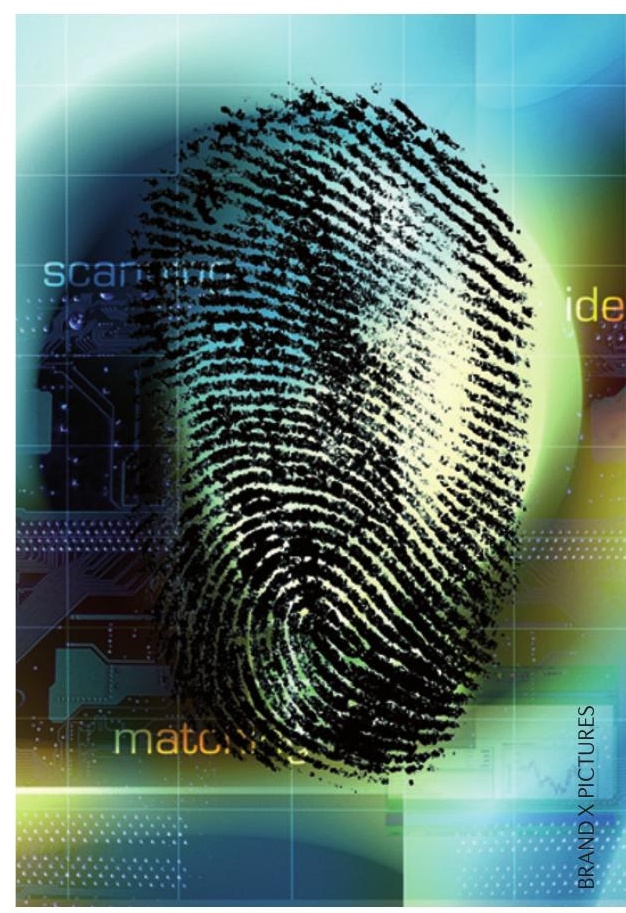

controls. Notably, both resveratrol and rolipram also increased levels of glucagon-like peptide 1, which has antidiabetogenic activities.

So, not only have Chung and colleagues identified a direct target of resveratrol and elucidated a biochemical pathway by which it confers metabolic benefits, they have also demonstrated that the PDE4 inhibitor rolipram reproduces all of these benefits. And with improved memory and protection against ageingrelated diseases having already been demonstrated for PDE4 inhibitors in animal models, these agents seem to have strong therapeutic potential. Katrin Legg

ORIGINAL RESEARCH PAPER Park, S.-J. et al. Resveratrol ameliorates aging-related metabolic phenotypes by inhibiting cAMP phosphodiesterases. Cell 148, 421-433 (2012) 